\title{
NOTAS CARIOSISTEMÁTICAS SOBRE FLORA ESPAÑOLA, I*
}

\author{
E. Valdés-Bermejo** \& J. Gómez García
}

\section{RESUMEN}

Se ha estudiado el número cromosómico de 21 táxones ibéricos. Creemos nuevos para la ciencia los recuentos siguientes: Gypsophila struthium Loefl. $(2 \mathrm{n}=34)$; Lepidium subulatum L. $(2 \mathrm{n}=16)$; Iberis cinerea Poiret $(2 \mathrm{n}=44)$; Erucastrum nasturtiifolium (Poiret) O. E. Schulz var. subbipinnatifidum (Lag.) Thell. $(2 \mathrm{n}=16)$; Teucrium gnaphalodes L'Hér. subsp. jaënnense (Lacaita) Rivas-Martínez $(2 \mathrm{n}=26)$; Teucrium pumilum L. $(2 \mathrm{n}=26)$; Centaurea alpina L. $(2 \mathrm{n}=30)$; Centaurea hyssopifolia Vahl $(2 \mathrm{n}=22)$; Launaea resedifolia (L.) O. Kuntzee $(2 \mathrm{n}=18)$.

Se reivindica el rango específico para Iberis cinerea Poiret y se insiste sobre la identidad de Centaurea linaresii Láz. Ibiza y C. alpina L.

\section{ABSTRACT}

The cromosome numbers of 21 Iberian taxa have been studied. The following numbers are, to our knowledge, new to science: Gypsophila struthium Loefl. $(2 \mathrm{n}=34)$; Lepidium subulatum L. $(2 \mathrm{n}=16)$; Iberis cinerea Poiret $(2 \mathrm{n}=44)$; Erucastrum nasturtiifolium (Poiret) O. E. Schluz var. subbipinnatifidum (Lag.) Thell. $(2 \mathrm{n}=16)$; Teucrium gnaphalodes L'Hér. subsp. jaënnense (Lacaita) Rivas-Martínez $(2 \mathrm{n}=26)$; Teucrium pumilum L. $(2 \mathrm{n}=26)$; Centaurea alpina L. $(2 \mathrm{n}=30)$; Centaurea hyssopifolia Vahl $(2 \mathrm{n}=22)$; Launaea resedifolia (L.) O. Kuntzee $(2 \mathrm{n}=18)$.

Specific rank is restored for Iberis cinerea Poiret, and the identity of Centaurea linaresii Láz. Ibiza and $C$. alpina $\mathrm{L}$. is remarked.

\section{INTRODUCCIÓN}

Con este título iniciamos una serie de notas en la que pretendemos ir publicando los números cromosómicos de plantas españolas.

Alguno de los datos que damos a conocer en este trabajo forman parte de un amplio estudio cariosistemático sobre la flora gipsícola española con

(*) Trabajo leído en el Simposio Conmemorativo del Centenario de Lagasca, celebrado en Sevilla del 30 de septiembre al 2 de octubre de 1976.

(**) Instituto Botánico A. J. Cavanilles, C.S.I.C. Plaza de Murillo, 2. Madrid-14. 
el que pretendemos conocer los orígenes, correlaciones y naturaleza de esta flora tan especializada.

A los números cromosómicos de los gipsófitos, añadimos otros, que sin serlo viven sin dificultad en estos medios y también aquellos de los que por no conocer datos cariológicos o ser recuentos realizados por vez primera en material español, consideramos de interés su publicación.

\section{MATERIAL Y MÉTODOS}

De todas las plantas estudiadas indicamos lugar de recolección, localización de los testigos, con el número de registro del Jardín Botánico de Madrid (JBM) cuando el material se conserva vivo y número del herbario (MA y MAF) cuando el material seco está allí depositado, y si es posible datos ecológicos y fitosociológicos.

La técnica empleada para visualización de los cromosomas ha sido la ya habitual en este tipo de trabajos: pretratamiento con 8-Hidroxiquinoleina, coloración con Orceina acética y aplastamiento. (E. Valdés-Bermejo, 1970).

\section{RESULTADOS}

Rumex obtusifolius L., Sp. Pl. 335 (1753)

2n=40. Pontevedra: entre Marín y Pardávila, lugares umbrosos ruderalizados. Leg. E. Valdés-Bermejo \& S. Castroviejo, 29-XII-1975. (JBM n. ${ }^{\circ}$ 000876).

Nuestro recuento coincide con el dado por numerosos autores, H. Kihara \& T. Ono (1926), H. W. Jansen (1936), A. Löve (1942), entre otros. Según nuestros datos, el único recuento anterior realizado en material español ha sido publicado por A. Löve \& E. Kjellqvist (1974) dando $2 n=40$ para una planta de Tragacete (Cuenca).

Herniaria fruticosa L., Cent. P1. 1:8 (1755) subsp. fruticosa

$2 n=18$ (fot. 1). Madrid: cerros yesíferos entre Aranjuez y Ontígola, en el Herniario-Teucrietum pumili Rivas-Martínez \& Costa, 1970. Leg. E. Valdés-Bermejo \& S. Castroviejo, 1-IV-1976. (JBM n. ${ }^{\circ}$ 1.662-76).

Gipsófito endémico español, diploide, como también lo son otras especies fruticosas del género ( $H$. fontanesii Gay). Nuestro número cromosómico coincide con el publicado por A. Lorenzo-Andreu \& P. García Sanz (1950), que estudiaron material aragonés. 
Gypsophila struthium Loefl., Iter Hisp. 79,303 (1758)

$2 n=34$ (fot. 2). Madrid: cerros yesíferos entre Aranjuez y Ontígola, $600 \mathrm{~m}$, en el Gypsophilo-Centaureetum hyssopipfoliae Rivas Goday, 1956. Leg. E. Valdés-Bermejo \& S. Castroviejo, 10-IX-1975. (JBM n. ${ }^{\circ}$ 0524-75).

Endemismo ibérico gipsícola (Castellano-Maestrazgo-Manchego, guadiciano-bacense, almeriense) del que no conocemos dato cariológico alguno. $2 \mathrm{n}=34$ es el número más frecuente y a la vez más bajo en género Gypsophila, se trata pues de una planta diploide.

Lepidium subulatum L., Sp. Pl. 644 (1753)

$2 n=16$ (fot. 3). Cuenca: Saceda del Río, Huete, cerros margoso-yesíferos oligocenos, $780 \mathrm{~m}$, en el Thymo-Ononidetum tridentatae Rivas-Martínez \& G. López, 1976. Leg. E. Valdés-Bermejo, S. Castroviejo \& J. Fernández Casas, 1-IV-1976. (JBM n. ${ }^{\circ}$ 1.670-76).

Endemismo gipsícola ibero-mauritano, se trata de una planta diploide cuyo número se da a conocer por vez primera.

Iberis cinerea Poiret, Encycl. Suppl. 3: 133 (1813)

=I. saxatilis L. subsp. cinerea (Poiret) P. W. Ball \& Heywood, Feddes Repert., 64: 62 (1961)

$=I$. subvelutina DC., Reg. Veg. Syst. Nat. 2: 397 (1821)

$2 n=44$ (fot. 4). Madrid: cerros yesíferos de los alrededores de Chinchón, $570 \mathrm{~m}$, en el Gypsophilo-Centaureetum hyssopifoliae Rivas Goday 1956. Leg. E. Valdés-Bermejo \& S. Castroviejo, 18-XI-1975. (JBM n. ${ }^{\circ} 1.026-$ 75).

No conocemos datos de recuentos anteriores para este taxon tetraploide. Además de los caracteres morfológicos, ecológicos y corológicos, el número cromosómico da mayor independencia específica a este taxon, que se había subordinado insistentemente al $I$. saxatilis $\mathrm{L}$.

Erucastrum nasturtiifolium (Poiret) O. E. Schulz, Bot. Jahrb. 54 Beibl., 119: 56 (1916)

var. nasturtiifolium.

$2 \mathrm{n}=16$ (fot. 5). Guadalajara: bordes de caminos.

Las plantas estudiadas se obtuvieron a partir de semillas cedidas por el Prof. Gómez-Campo y figuran en su "Colección de germoplasma de Cruciferas" con el n..$^{\circ}$ 1.220-67. Nuestro recuento confirma las observaciones de L. de A. Coutinho \& A. Lorenzo-Andreu (1948) y Harberd (1972). Este 
último autor y Manton (1932) señalan la existencia de una raza tetraploide $(\mathrm{n}=16)$ de la que no indican procedencia.

Erucastrum nasturtiifolium (Poiret) B. O. Schulz var. subbipinnatifidum (Lag.) Thell.

2n=16 (fot. 6). León: al norte de los Montes de León.

Plantas que proceden de semillas y que figuran en la "Colección de Germoplasma” del Prof. Gómez-Campo con el n. ${ }^{0}$ 1.981-71. Se trata de un taxon diploide y al parecer es la primera vez que se estudia el número cromosómico de este taxon.

Cistus clusii Dunal in DC., Prodr. 1: 266 (1824)

2n=18. Madrid: Ciempozuelos, cerros del Butarrón, $650 \mathrm{~m}$, en el Cisto-Rosmarinetum Rivas-Martínez \& Izco in Izco, 1969. Leg. E. ValdésBermejo, S. Castroviejo \& M. Costa, 18-XI-1975. (JBM n. ${ }^{\circ}$ 1.020-75).

Es la primera vez que se estudia material peninsular. Nuestro resultado confirma el publicado para las Baleares por Dahlgren \& al. (1971).

Helianthemum hirtum (L.) Miller, Gard. Dict. ed. 8, n. ${ }^{\circ} 14$ (1768)

$2 n=20$ (fot. 7). Madrid: Aranjuez, cerros yesíferos en las proximidades del mar de Ontígola. En el Gypsophilo-Centaureetum hyssopifoliae Rivas Goday, 1956. Leg. J. Gómez García \& E. Valdés-Bermejo, 10-V-1973. (MAF).

Primera vez que se estudia material de procedencia ibérica. Nuestro número coincide con el conocido con anterioridad para el Norte de Africa (Reese, 1957).

Helianthemum squamatum (L.) Pers., Syn. Pl. 2: 78 (1806)

$2 n=10$ (fot. 8). Madrid: cerros yesíferos entre Aranjuez y Ontígola, $600 \mathrm{~m}$, en el Gypsophilo-Centaureetum hyssopifoliae Rivas-Goday, 1956. Leg. E. Valdés-Bermejo \& S. Castroviejo, 10-XI-1975. (JBM n. ${ }^{\circ}$ 0518-75).

Gypsófito endémico iberomauritano. Su número cromosómico es el más bajo encontrado hasta ahora en el género. Nuestros resultados coinciden con los de L. de A. Coutinho \& A. Lorenzo-Andreu (1948) que estudiaron esta planta de los yesos de Aragón. Se trata de un diploide paleoendémico. (Cl. Favarger \& J. Contandriopoulos, 1961). 
Frankenia thymifolia Desf., Fl. Atl. 1: 316 (1798)

$2 n=10$. Madrid, parte basal de los cerros margoso-yesíferos, entre Aranjuez y Ontígola, $500 \mathrm{~m}$, en el Artemisio-Frankenietum thymifoliae Rivas-Martínez \& Izco, 1972. Leg. E. Valdés-Bermejo \& S. Castroviejo, 10-XII-1975. (JBM n. ${ }^{\circ}$ 0534-75).

Elemento mediterráneo gipsonitrófilo, diploide, estudiado por vez primera en material español. Para plantas del norte de Africa, G. Reese (1957) da $2 n=30$ cromosomas.

Teucrium capitatum L., Sp. Pl. 2: 566 (1753) subsp. capitatum

2n=26 (fot. 9). Madrid: Aranjuez, cerros yesíferos, 600 m, en el Gypsophilo-Centaureetum hysopifoliae Rivas Goday, 1956. Leg. E. ValdésBermejo \& S. Castroviejo, 10-XI-1975. (JBM n. ${ }^{\circ}$ 0520-75).

Nuestros resultados confirman los de S. Puech (1972), L. B. Maleci \& B. Mori (1972) y M. Guinochet \& M. Lefrant (1972). Se trata pues de una población diploide. Recientemente se ha descubierto en Los Barrios (Toledo) una población tetraploide. (S. Puech, 1975).

\section{Teucrium gnaphalodes L'Hér.}

subsp. jaënnense (Lacaita) Rivas-Martínez, Candollea 31: 114 (1976).

$2 n=26$ (fot. 10). Madrid: Aranjuez, parte basal de los cerros yesíferos $550 \mathrm{~m}$, en el Artemisio-Frankenietum thymifoliae Rivas-Martínez \& Izco, 1972. Leg. E. Valdés-Bermejo \& S. Castroviejo, 10-XI-1975. (JBM n. 053375).

Endemismo ibérico basifilo, diploide. Nuestras observaciones confirman las de S. Puech (1974) aunque este autor no hace referencia a la subespecie estudiada. La subsp. jaënnense se distingue por sus flores blanquecinas y sus tricomas blanco amarillentos. (S. Rivas-Martínez, 1976: 114).

Teucrium pumilum L., Cent. Pl. 1: 115 (1755) subsp. pumilum

$2 \mathrm{n}=26$ (fot. 11). Cuenca: Saceda del Río, cerros yesíferos, $780 \mathrm{~m}$. Leg. E. Valdés-Bermejo \& S. Castroviejo, 1-IV-1976. (JBM n. ${ }^{0}$ 1.660-76). Madrid: Aranjuez, cerros yesíferos entre Aranjuez y Ontígola, en el Herniario-Teucrietum pumili Rivas-Martínez \& Costa, 1970. Leg. E. ValdésBermejo \& J. Gómez García, 10-V-1973. MAF. 
Gipsofito endémico presente en los sectores manchego y guadicianobacense (Rivas-Martínez, 1974) de la península ibérica. Especie diploide que no había sido estudiada cariológicamente.

Centaurea alpina L., Sp. Pl. 910 (1753)

=C. Linaresii Láz.-Ibiza, Anal. Soc. Esp. Hist. Nat. 29: 152-155 (1900)

$2 n=30$ (fot. 12). Cuenca en los alrededores del Balneario de Solán de Cabras. Suelo calcáreo, $1.000 \mathrm{~m}$. Leg. E. Valdés-Bermejo, S. Castroviejo \& J. Fdez. Casas, 1-IV-1976. (JBM n. ${ }^{\circ}$ 1.689-76).

Según la bibliografía por nosotros consultada no se conoce ningún dato sobre el número cromosómico de esta planta. Siendo $\mathrm{x}=15$ el número básico de la sect. Centaurium Cass. la planta que estudiamos es diploide y presenta dos parejas de cromosomas satelíferos de distinto tamaño.

Opinamos que Centaurea alpina L. y C. linaresii Láz. - Ibiza deben considerarse sinónimos ya que los caracteres que las separarían no parecen constantes. Sobre su identidad ya opinaron F. Bellot \& M. E. Ron (1975).

Centaurea hyssopifolia Vahl, Symb. Bot. 1: 75 (1790)

$2 \mathrm{n}=22$. (fot. 13). Madrid: Aranjuez, cerros yesíferos, $600 \mathrm{~m}$, en el Gypsophilo-Centaureetum hyssopifoliae Rivas Goday, 1956. Leg. E. ValdésBermejo \& S. Castroviejo. 30-XII-1975. (JBM n. ${ }^{\circ}$ 0047-76).

Es la primera vez que se estudia el número cromosómico de este gipsófito endémico manchego. Se trata de un taxon diploide cuyo número haploide de cromosomas $\mathrm{n}=11$ coincide con el número básico $(\mathrm{x}=11)$ de toda la Sect. Lepteranthus (DC.) Dumort. del género Centaurea $L$. donde se la incluye.

Centaurea limbata Hoffmanns. \& Link, Fl. Port. 2:221 (1820-1828).

$2 n=18$ (fot. 14). La Coruña: Finisterre, acantilados junto al faro. En comunidades litorales de Ulex europaeus subsp. latebracteatus, $80 \mathrm{~m}$. Leg. E. Valdés-Bermejo \& S. Castroviejo, 30-XII-1975. (JBM no. 0047-76).

Endemismo del noroeste de la península ibérica, diploide. Es la primera vez que se estudia en material español, encontrando el mismo número cromosómico que el publicado para el norte de Portugal por A. Fernandes \& M. Queiros (1971). Cromosonas relativamente grandes y una pareja de satelíferos. Su número haploide $\mathrm{n}=9$ es el básico $(\mathrm{x}=9)$ de la Sect. Paniculatae (Hayek) Dostál, a la que pertenece. 
Artemisia herba-alba Asso, Syn. Stirp. Arag. 117 (1779). var. herba-alba

=A. herba-alba Asso var. incana Boiss. Voy. Bot. Midi Esp. 2:323 (1839). $=A$. aragonensis Lam. in Gren. \& Godron, Fl. Fr. 2:156 (1850).

$2 \mathrm{n}=18$ (fot. 15). Madrid: parte basal de los cerros margoso-yesíferos entre Aranjuez y Antígola, $580 \mathrm{~m}$, en el Artemisio-Frankenietum thymifoliae. Rivas-Martínez \& Izco, 1972. Leg. E. Valdés-Bermejo \& S. Castroviejo, 10-XI-1975 (JBM no. 0544-75).

Es la primera vez que se estudia el número cromosómico en material español y resulta ser diploide. T. Kawatani \& T. Ohno (1964) y A. Murin \& I. I. Chaudhri (1970) dan $2 n=36$ para plantas no españolas.

Launaea resedifolia (L.) O. Kuntzee, Revis. Gen. 1:351 (1891).

$2 \mathrm{n}=18$. Madrid: cerros yesíferos entre Aranjuez y Ontígola, $600 \mathrm{~m}$, en el Gypsophilo-Centaureetum hyssopifoliae. Rivas Goday, 1956. Leg. E. Valdés-Bermejo \& S. Castroviejo, 10-XI-1975. (JBM no. 0523-75).

Es la primera vez que se da a conocer el número cromosómico de esta planta. Se trata de un taxon diploide, con número básico $\mathrm{x}=9$.

Lapiedra martinezii Lag., Gen. Sp. 14 (1816).

$2 n=22$. (fot. 16 y 17). Málaga: Carratraca, Sierra de Caparaín, fisuras de rocas dolomitícolas. Leg. G. López González, IV-1976 (JBM no. 1396-75).

Nuestros resultados coinciden con los de A. Fernandes (1950), T. W. J. Gadella \& al. (1966), B. Valdés (1970) y M. Ruiz Rejón (1974). Los tres últimos autores estudian material de Alicante, Murcia y Granada, respectivamente.

Narcissus papyraceus Ker-Gawler, Bot. Mag. 24 :t. 947 (1806).

$2 n=22$. Badajoz: cunetas de carretera, de Cabeza del Buey a Talarrubias. Leg. J. L. Pérez Chiscano, 7-IV-1971. MAF 78859.

Aunque este número es la confirmación de varios recuentos previos para esta especie (A. Fernandes, 1937, 1967-68, 1971 y S. Weitz \& N. Feibrun, 1972), parece ser la primera vez que se estudia en material español.

Esta planta, que con frecuencia se subordina a $N$. tazetta, la creemos merecedora del rango específico. El color de las flores y el número cromosómico $(2 \mathrm{n}=20$ para $N$. tazetta) son, entre otras, buenas diferencias. 
TABLA CON LOS RESULTADOS OBTENIDOS

\begin{tabular}{|c|c|c|}
\hline & $2 \mathrm{n}$ & $\begin{array}{c}2 \mathrm{n} \\
\text { Determinado por } \\
\text { otros autores }\end{array}$ \\
\hline Rumex obtusifolius & 40 & 40 \\
\hline Herniaria fruticosa & & \\
\hline subsp. fruticosa & 18 & 18 \\
\hline Gypsophila struthium .... & 34 & \\
\hline Lepidium subulatum ... & 16 & \\
\hline Iberis cinerea .................... & 44 & \\
\hline Erucastrum nasturtiifolium & & \\
\hline var. nasturtiifolium ........... & 16 & 16,32 \\
\hline var. subbipinnatifidum ..... & 16 & \\
\hline Cistus clusii ................. & 18 & 18 \\
\hline Helianthemum hirtum & 20 & 20 \\
\hline , squamatum & 10 & 10 \\
\hline Frankenia thymifolia .............. & 10 & 30 \\
\hline Teucrium capitatum & & \\
\hline $\begin{array}{l}\text { subsp. capitatum } \ldots \ldots \ldots \ldots \ldots \ldots \ldots \ldots \ldots \ldots \ldots \ldots \ldots \ldots \ldots \ldots \ldots \\
\text { Teucrium gnaphalodes }\end{array}$ & 26 & 26,52 \\
\hline 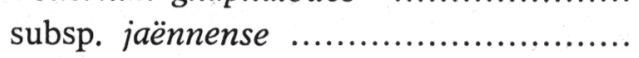 & 26 & \\
\hline $\begin{array}{l}\text { Teucrium pumilum ............................... } \\
\text { subsp. pumilum }\end{array}$ & 26 & \\
\hline Centaurea alpina & 30 & \\
\hline , hyssopifolia .... & 22 & \\
\hline , limbata & 18 & 18 \\
\hline Artemisia herba-alba ....................... & 18 & 36 \\
\hline Launaea resedifolia ............................ & 18 & \\
\hline Lapiedra martinezii ............................ & 22 & 22 \\
\hline Narcissus papyraceus ......................... & 22 & 22 \\
\hline
\end{tabular}




\section{BIBLIOGRAFIA}

Bellot, F. \& Ron, M. E. 1975. Notas sobre algunas Centaureas de España. Trab. Dep. Botánica, Madrid 7:3-9.

Coutinho, L. DE A. \& Lorenzo-Andreu, A. 1948. Contribución al estudio cariológico de la flora espontánea de la estepa de Aragón, I. Anal. Est. Exp. Aula Dei $1: 3-13$.

Dahlgren, R. Karlsson, Th. \& Larsen, P. 1971. Studies on the Flora of the Balearic Islands, I. Bot. Notiser 124:249-269.

Dostál, J. 1976. Centaurea L. In: T. G. Tutin \& al. Flora Europaea 4:254-301. Cambridge.

Favarger, Cl. \& Contandriopoulos, J. 1961. Essai sur l'endemisme. Bull. Soc. Bot. Suisse $71: 384-407$.

FERNANDES, A. 1937. Le problème de Narcissus tazetta L., I. Les formes à 22 chromosomes somatiques. Bol. Soc. Broteriana 12:159-219.

Fernandes, A. 1950. Sur la caryologie de Lapiedra martinezii Lag. Bol. Soc. Broteriana $24: 281-319$.

Fernandes, A. 1967-68. Contribution a la connaissance de la biosystématique de quelques especes du genre Narcissus L. Portugalia Act. Biol. (Sér. B) $9: 1-44$.

Fernandes, A. \& Queiros, M. 1971. Sur la caryologie de quelques plantes récoltees pendant la III Reunion de Botanique Peninsulaire. Men. Soc. Broteriana $21: 343-379$.

Fernandes, A. \& Queiros, M. 1971. Contribution à la connaissance cytotaxinomique des spermatophyta du Portugal, II. Compositae. Bol. Soc. Broteriana $45: 5-121$

Gadella, T. W. J., Kliphuis, E. \& Mennega, E. A. 1966. Chromosome numbers of some flowering plants of Spain and S. France. Act. Bot. Neerlandica $15: 484-489$.

Guinochet, M. \& Lefranc, M. 1972. IOPB chromosome number reports XXXVII. Taxon $21: 495-500$.

Herberd, D. J. 1972. A contribution to the cytotaxonomy of Brassica (Cruciferae) and its allies. Bot. J. Linn. Soc. 65:1-23.

Jansen, H. W. 1936. Meiosis in Rumex. I. Polyploidy and the origen of new species. Cytologia $7: 1-22$.

Kinara, H. \& Ono, T. 1926. Chromosomenzahlen and Systematische Gruppierung der Rumex-Arten. Zeitschr. Zellforsch. u mikrosk. Anat. 4:475-481.

Kawatani, T. \& Ohno, T. 1964. Chromosome numbers in Artemisia. Bull. Nat. Inst. Hygienic Sci. 82:183-193.

Lorenzo-Andreu, A. \& García Sanz, P. 1950. Cromosomas somáticos de plantas espontáneas en la estepa de Aragón, II. Anal. Est. Exp. Aula Dei 2:12-20.

Löve, A. 1942. Cytogenetic studies in Rumex, III. Some notes on Scandinavian species of the genus. Hereditas 28:289-296.

Löve, A. \& KJellqvist, E. 1974. Cytotaxnomy of Spanish plants, III. Dicotyledons: Salicaceae-Rosaceae. Lagascalia, 4:3-32.

Maleci, L. B. \& MoRi, B. 1972. Numeri cromosomici per la flora italiana. Inform. Bot. Ital. 4: 224-236. 
Manton, I. 1932. Introduction to the general cytology of the Cruciferae. Ann. Bot. London 46:509-556.

Murin, A. \& Chaudhri, I. I. 1970. IOPB Chromosome number reports, XXVII. Taxon 19:264-269.

PUEch, S. 1970. IOPB Chromosome number reports XXVII. Taxon 19:437-442.

PUech, S. 1972. IOPB Chromosome number reports, XXXVII. Taxon 21:495-500.

Puech, S. 1974. IOPB Chromosome number reports, XLVI. Taxon 23:810.

PuEch, S. 1975. Contibution caryologique à l'etude des Teucrium de la Secttion Polium du bassin méditerranéen occidental. Colloques Intern. CNRS, n. ${ }^{\circ}$ 225: La flore du bassin méditerranéen: 223-238.

Rivas-Martínez, S. 1974. Avance sobre una síntesis corológica de la península ibérica, Baleares y Canarias. Anal. Inst. Bot. Cavanilles 30:69-87.

Rivas-Martfnez, S. 1974. Sobre el Teucrium pumilum L. (Labiatae) y sus especies afines. Anal. Inst. Bot. Cavanilles 31:79-96.

Rivas-Martínez, S. 1976. De plantis Hispaniae notulae systematicae, chorologicae et ecologicae, I. Candollea 31:111-116.

Ruiz Rejón, M. 1974. IOPB Chromosome number reports, XLVI. Taxon 23:805-806.

Reese, G. 1957. Uber die Poyploidiespektren in der nordsaherischen Wüstenpflanzen. Flora 144:598-634.

VALDÉs, B. 1970. Números cromosómicos de algunas plantas españolas. Bol. $R$. Soc. Española Hist. Nat. (Biol.) 68:193-197.

VALDÉs-Bermejo, E. 1970. Estudios cariológicos en crucíferas españolas de los géneros Moricandia DC., Vella L., Carrichtera Adans. y Hutera Porta. Anal. Inst. Bot. Cavanilles 27:125-133.

Weitz, S. \& Feinbrun, N. 1972. Cytology and Systematics of Narcissus tazetta L. in Israel. J. Bot. (Israel) 21:9-20.

(Recibido el 6 de noviembre de 1976) 

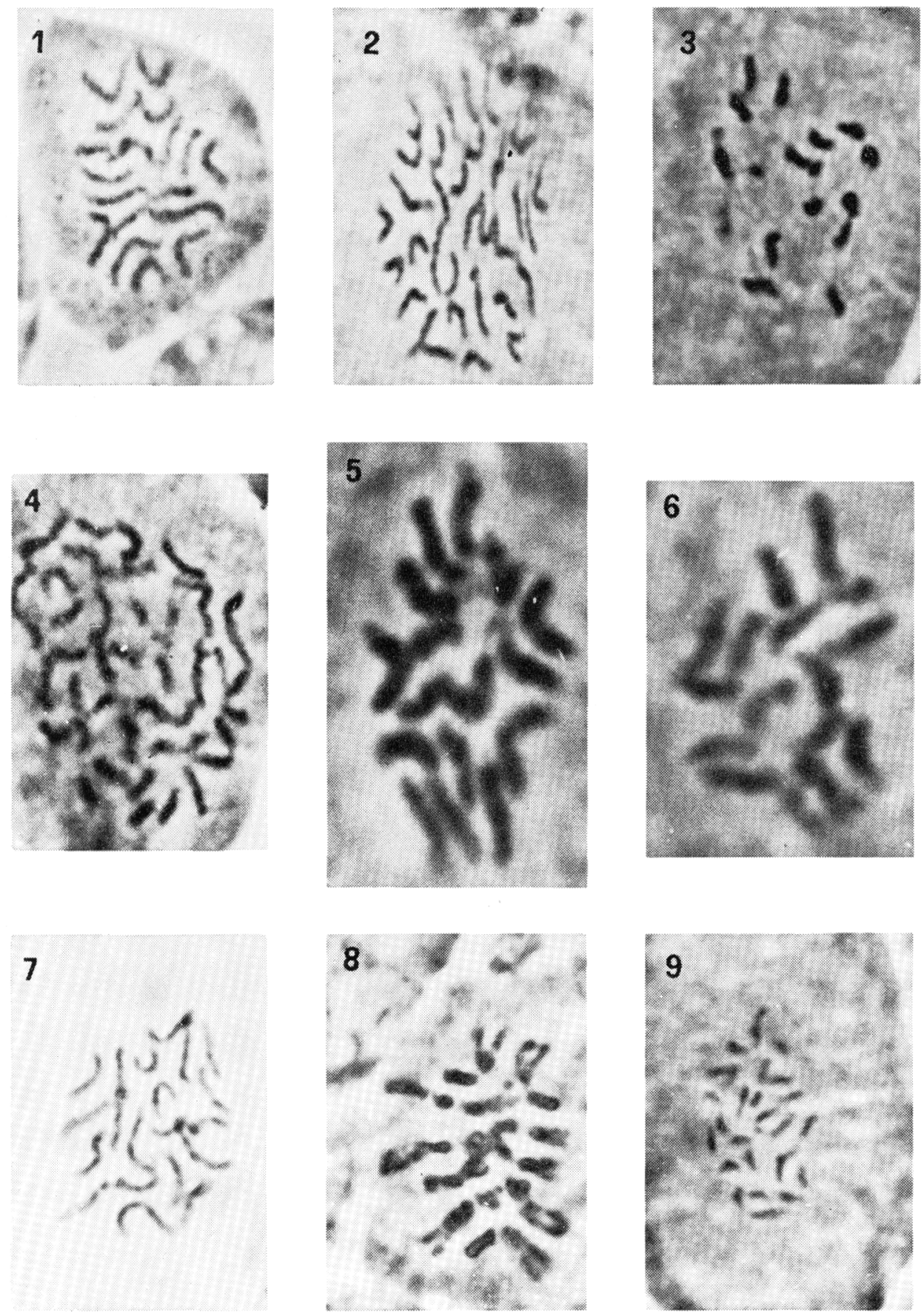

Metafases somáticas de las plantas estudiadas

1. Herniaria fruticosa subsp. fruticosa, $2 n=18(\times 1.500)$; 2. Gypsophila struthium, $2 \mathrm{n}=34(\times 1.500) ; 3$. Lepidium subulatum, $2 \mathrm{n}=16(\times 1.500) ; 4$. Iberis cinerea, $2 \mathrm{n}=44(\times 1.500) ; 5$. Erucastrum nasturtiifolium var. nasturtiifolium, $2 \mathrm{n}=16$ $(\times 2.000)$. 6. Erucastrum nasturtiifolium var. subbipinnatifidum, $2 \mathrm{n}=16(\times 2.000)$; 7. Helianthemun hirtum, $2 \mathrm{n}=20(\times 1.500) ; 8$. Helianthemun squamatum, $2 \mathrm{n}=10$ $(\times 1.500) ; 9$. Teucrium capitatum subsp. capitatum, $2 \mathrm{n}=26(\times 1.500)$. 

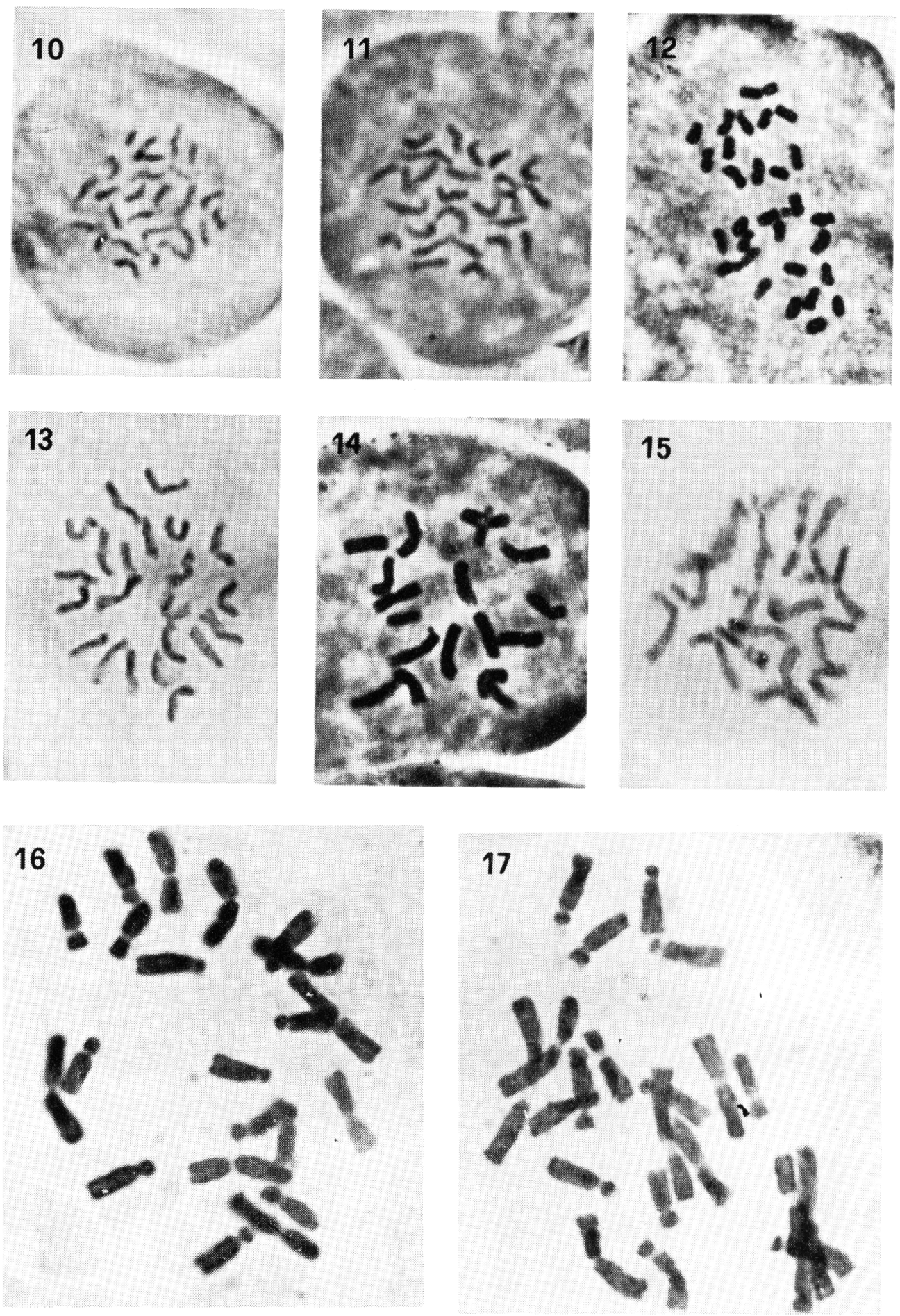

Metafases somáticas de las plantas estudiadas

10. Teucrium gnaphalodes subsp. jaënnense, $2 \mathrm{n}=26(\times 1.500) ; 11$. Teucrium pumilum, $2 \mathrm{n}=26(\times 1.500)$. 12. Centaurea alpina, $2 \mathrm{n}=30(\times 1.500) ; 13$. Centaurea hyssopifolia, $2 \mathrm{n}=22(\times 1.500) ; 14$. Centaurea limbata, $2 \mathrm{n}=18(\times 1.500) ; 15$. Artemisia herba-alba, $2 \mathrm{n}=18(\times 1.500) ; 16$ y 17 . Lapiedra martinezii, $2 \mathrm{n}=2(\times 2.000)$. 